\title{
Thermotolérance des Candida
}

\section{Considérations écologiques, pathologiques et taxonomiques}

\author{
par H. SAEZ, F. TRAORE et M. THERIZOL \\ Laboratoire d'Ethologie du Muséum, Parc zoologique, \\ 53, avenue de Saint-Maurice, F 75012 Paris \\ Groupe de Mycologie fondamentale et appliquée de l'Université de Lille.
}

\section{Résumé.}

La température maximale de développement a été recherchée chez 477 souches de Candida appartenant à 23 espèces et 1 variété, dont quelques espèces actuellement rangées ailleurs que dans les Candida mais bien connues, sous ce nom générique, en mycologie médicale et vétérinaire. $C$. pseudotropicalis est l'espèce la plus thermotolérante $\left(49^{\circ}\right), C$. albicans n'arrivant qu'en deuxième position $\left(42^{\circ}-48^{\circ}\right)$; l'espèce la moins thermotolérante, dans notre classement, est $C$. humicola $\left(29^{\circ}\right)$, mais la souche la moins thermotolérante est un $C$. sake $\left(26^{\circ}-32^{\circ}\right)$. Des considérations écologiques, pathologiques et taxonomiques sont rapportées. Dans le groupe $C$. krusei une relation entre la température maximale et l'assimilation d'un sucre a été constatée, laissant présager une liaison plus intime entre la caractéristique thermique et l'équipement enzymatique d'une levure.

\section{Summary.}

Thermotolerance of the Candida. Relationships with ecology, pathology and taxonomy.

An investigation on the maximum temperature of growth is reported on 477 strains of Candida, 23 species and 1 variety, including species actually not identified as Candida, but well known under that generic name in medical and veterinary mycology. C. pseudotropicalis is the most thermotolerante species $\left(49^{\circ}\right), C$. albicans coming only in second position $\left(42^{\circ}-48^{\circ}\right)$; the less thermotolerante species is C. humicola $\left(29^{\circ}\right)$, but the less thermotolerante strain is a $C$. sake $\left(26^{\circ}-32^{\circ}\right)$. The relationships between the maximum temperature of growth and the ecology, the pathology and the taxonomy are discuted. In 
the group $C$. krusei a linkage between the maximum temperature and the utilisation of a sugar has been established, auguring a more close relation between the thermic characteristic and the enzymologic equipment of a yeast.

Dans la monographie des levures de 1970 (4) une caractéristique physiologique nouvelle est signalée, dans la description de quelques espèces seulement, la température maximale de développement. Van Uden a été l'un des premiers à indiquer systématiquement la limite thermique des espèces décrites. Avec ses collaborateurs, Do Carmo-Sousa, Farinha, il a utilisé cette propriété dans des questions de taxonomie et de pathogénéicité (13), (14).

Depuis une dizaine d'années nous recherchons aussi la température maximale des levures isolées ou reçues au laboratoire. Les résultats que nous rapportons, ici, concernent uniquement les espèces du genre Candida. Sur les 477 souches examinées, 5 proviennent de collections, les autres ont été testées aussitôt après isolement de leur habitat coutumier ou fortuit: le tube digestif, principalement, ou tout autre endroit de l'organisme humain et animal.

Important par le nombre d'entités qu'il renferme, le genre Candida l'est davantage, pour les organismes à sang chaud, sur le plan écologique et parasitologique. Comme tous les genres des Cryptococcacées, il accueille des levures anascosporées dont certaines, au grès des recherches et parfois aussi de la chance de l'investigateur, sont reclassées ailleurs quand une forme de sexualité a été mise en évidence. C'est un genre d'attente, en continuelle mouvance.

Parmi les 24 espèces et variété entre lesquelles se répartissent nos 477 souches, quelques-unes ne sont plus, actuellement, des Candida. Nous avons conservé leur nom générique, soit parce que certaines découvertes demandent encore confirmation, soit parce qu'il s'agit de binomes depuis fort longtemps utilisés en mycologie médicale et vétérinaire et dont les nouvelles appellations nécessitent un temps d'adaptation.

Pour C. albicans, transferré dans le genre Syringospora Quinquad emend., Van Der Walt (12) a d'ailleurs conservé l'épithète «albicans»; dans ce genre l'auteur a encore rangé deux autres Candida voisins: $C$. stellatoidea et $C$. claussenii. Leurs travaux sur la sexualité ont conduit Pitt \& Miller (6) (7), à classer C. pulcherrima et C. reukaufii (3) dans le genre Metschnikowia qui ne réunissait, jusque-là, que des levures à habitat aquatique. Auparavant, Wickerham (in 4) avait fait de ces deux espèces des Chlamydozyma. Les «mating-types » de C. guilliermondii sont identifiés à Pichia guilliermondii. C. bovina Van Uden et Do Carmo-Sousa, que Kreger-Van Rij considère comme la forme imparfaite de Saccharomyces telluris Van Der Walt (in 4), est passé dans le genre Torulopsis.

Les levures sont identifiées au laboratoire selon les techniques classiques (observation des éléments morphologiques et des propriétés physiologiques: fermentation, assimilation des produits hydro-carbonés et du nitrate de potassium); l'action sur le tétrazolium, d'autres tests enzymologiques peuvent venir s'ajouter à ces techniques. Il en va de même pour toutes les souches de $C$. albicans réunies pour ce travail, lesquelles 
n'ont jamais été différenciées uniquement à partir d'une méthode de diagnose rapide (obtention précoce du chlamydospores, de tubes germinatifs, etc.).

Pour la plupart des micromycètes saprophytes ou parasites de l'homme, des mammifères et des oiseaux, nous recherchons la température maximale de développement, au cours d'un premier temps, selon une technique standard: nous ne faisons varier qu'un seul paramètre, la température d'incubation, laquelle est élevée d'un degré centigrade à chaque nouvelle exposition. Ce sont les résultats ainsi obtenus que nous rapportons pour les Candida. Le milieu nutritif est toujours une gélose de Sabouraud (peptone $1 \%$; glucose $2 \%$ ) ajustée à $\mathrm{pH} 6,9$. Des tubes de $16 \times 160$ sont ensemencés à partir d'une jeune culture obtenue sur le même milieu ci-dessus. Les tubes sont incubés à l'obscurité et conservés au moins 15 jours à un niveau thermique donné.

\section{Résultats}

C. albicans: 232 souches testées, dont 25 isolées à l'état parasitaire et 2 souches de collection, les sérotypes A et B reçus du Laboratoire de Parasitologie de la Faculté de Médecine de Lyon. Température maximale:

a) Ensemble des souches:

$\begin{array}{rrr}-42^{\circ}: & 1 \text { souche; } & -46^{\circ}: 122 \text { souches; } \\ -44^{\circ}: & 5 \text { souches; } & -47^{\circ}: 59 \text { souches; } \\ -45^{\circ}: & 42 \text { souches; } & -48^{\circ}: 3 \text { souches; }\end{array}$

b) Souches isolées à l'état parasitaire :

$-44^{\circ}: 1$ souche; $\quad-46^{\circ}: 13$ souches;

$-45^{\circ}: 6$ souches; $\quad-47^{\circ}: 4$ souches;

$-48^{\circ}: 1$ souche.

c) Souches de collection :

$-45^{\circ}$ : sérotype B ; $\quad-46^{\circ}$ : sérotype A;

Température maximale de la majorité des souches de C. albicans: $46^{\circ}$.

C. bovina: 1 souche testée. Température maximale: $46^{\circ}$.

C. diversa; 1 souche testée. Température maximale : $37^{\circ}$.

C. guilliermondii : 11 souches testées. Température maximale:

$-39^{\circ}: 4$ souches; $\quad-42^{\circ}: 2$ souches;

$-40^{\circ}: 3$ souches; $\quad-43^{\circ}: 1$ souche;

$-44^{\circ}: 1$ souche.

Température maximale de la majorité des souches : $39^{\circ}$. 
C. humicola: 1 souche testée. Température maximale: $29^{\circ}$.

C. intermedia: 1 souche testée. Température maximale: $37^{\circ}$.

C. krusei : 40 souches testées, dont 1 souche de collection: C.B.S., $\mathrm{n}^{\circ} 573$, reçue de Delft, Hollande. Température maximale:

a) Ensemble des souches :

$-41^{\circ}: 1$ souche; $\quad-45^{\circ}: 12$ souches;

$-44^{\circ}$ : 2 souches; $\quad-46^{\circ}: 20$ souches;

$-47^{\circ}: 5$ souches.

b) Souche de collection :

$-44^{\circ}$ : souche C.B.S. $\mathrm{n}^{\circ} 573$.

Température maximale de la majorité des souches: $46^{\circ}$.

C. lambica : 48 souches testées, dont 2 souches de collection: Mycoderma lambica C.B.S. $\mathrm{n}^{\circ} 1876$ et Torula monoso C.B.S. $\mathrm{n}^{\circ}$ 603, reçues de Delft, Hollande. Température maximale :

a) Ensemble des souches:

$-35^{\circ}: 1$ souche; $\quad-39^{\circ}: 20$ souches;

$-37^{\circ}: 5$ souches; $\quad-40^{\circ}: 3$ souches;

$-38^{\circ}: 16$ souches; $\quad-41^{\circ}: 3$ souches.

b) Souches de collection :

$-35^{\circ}$ : Mycoderma lambica $-37^{\circ}$ : Torula monosa C.B.S. $\mathrm{n}^{\circ} 603$.

C.B.S. $\mathrm{n}^{\circ} 1876$;

Température maximale de la majorité des souches : $39^{\circ}$.

C. lipolytica: 12 souches testées. Température maximale :

$-27^{\circ}: 1$ souche; $\quad-32^{\circ}: 4$ souches;

$-28^{\circ}: 1$ souche; $\quad-33^{\circ}: 1$ souche;

$-31^{\circ}: 2$ souches; $\quad-37^{\circ}: 3$ souches.

Température maximale de la majorité des souches: $32^{\circ}$.

C. lipolytica variété deformans : 3 souches testées. Température maximale :

$-32^{\circ}: 1$ souche; $\quad-33^{\circ}: 1$ souche;

$-34^{\circ}: 1$ souche.

C. lusitaniae: 4 souches testées. Température maximale :

$-42^{\circ}: 1$ souche; $\quad-45^{\circ}: 1$ souche;

$-44^{\circ}: 1$ souche; $\quad-46^{\circ}: 1$ souche. 
C. nouvelii : 2 souches testées. Température maximale :

$-46^{\circ}: 2$ souches.

C. obtusa: 1 souche testée. Température maximale: $45^{\circ}$.

C. parapsilosis : 12 souches testées. Température maximale:

$-38^{\circ}: 1$ souche; $\quad-42^{\circ}: 3$ souches;

$-40^{\circ}: 1$ souche; $\quad-43^{\circ}: 3$ souches;

$-41^{\circ}: 1$ souche; $\quad-44^{\circ}: 3$ souches.

C. pseudotropicalis : 1 souche testée. Température maximale : $49^{\circ}$.

C. pulcherrima: 6 souches testées. Température maximale:

$-37^{\circ}: 2$ souches; $\quad-38^{\circ}: 4$ souches.

Température maximale de la majorité des souches: $38^{\circ}$.

C. ravautii : 4 souches testées. Température maximale:

$-39^{\circ}: 2$ souches; $\quad-40^{\circ}: 1$ souche; $-41^{\circ}: 1$ souche.

Température maximale de la majorité des souches : $39^{\circ}$.

C. rugosa: 4 souches testées. Température maximale:

$-37^{\circ}: 1$ souche; $\quad-38^{\circ}: 2$ souches;

$-42^{\circ}: 1$ souche.

Température maximale de la majorité des souches : $38^{\circ}$.

C. sake: 42 souches testées. Température maximale :

$-26^{\circ}: 1$ souche; $\quad-30^{\circ}: 12$ souches;

$-28^{\circ}: 5$ souches; $\quad-31^{\circ}: 9$ souches;

$-29^{\circ}: 12$ souches; $\quad-32^{\circ}: 3$ souches.

Température maximale de la majorité des souches: 29-30.

C. salmanticensis : 1 souche testée. Température maximale: $37^{\circ}$.

C. tropicalis: 31 souches testées. Température maximale :
$-42^{\circ}: 5$ souches;
$-45^{\circ}: 10$ souches;
$-43^{\circ}: 2$ souches;
$-46^{\circ}: 8$ souches;
$-44^{\circ}: 4$ souches;
$-47^{\circ}: 2$ souches.

Température maximale de la majorité des souches : $45^{\circ}$. 
C. valida (ex. C. mycoderma pro parte): 1 souche testée. Température maximale : $43^{\circ}$.

C. vartiovaarai : 1 souche testée. Température maximale: $32^{\circ}$.

C. zeylanoides : 11 souches testées. Température maximale:

$\begin{array}{ll}-32^{\circ}: 4 \text { souches; } & -34^{\circ}: 2 \text { souches; } \\ -33^{\circ}: 2 \text { souches; } & -35^{\circ}: 2 \text { souches; }\end{array}$

$-36^{\circ}: 1$ souche.

Température maximale de la majorité des souches : $32^{\circ}$.

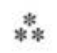

Selon l'ordre décroissant des températures maximales on peut ainsi ranger ces 24 Candida :

1. C. pseudotropicalis ...... $49^{\circ}$

2. C. albicans ............ $42^{\circ}-48^{\circ}$

13. C. ravautii ............. $39^{\circ}-41^{\circ}$

3. C. tropicalis ........... $42^{\circ}-47^{\circ}$

14. C. lambica ............. $35^{\circ}-41^{\circ}$

4. C. krusei ............ $41^{\circ}-47^{\circ}$

15. C. pulcherrima $\ldots \ldots \ldots \ldots, 37^{\circ}-38^{\circ}$

5. C. bovina ............ $46^{\circ}$

16. C. diversa $\ldots \ldots \ldots \ldots \ldots .37^{\circ}$

C. nouvelii .............. $46^{\circ}$

7. C. lusitaniae $\ldots \ldots \ldots \ldots \ldots 42^{\circ}-46^{\circ}$

8. C. obtusa ........... $45^{\circ}$

9. C. guilliermondii ........ $39^{\circ}-44^{\circ}$

10. C. parapsilosis ........... $38^{\circ}-44^{\circ}$

11. C. valida $\ldots \ldots \ldots \ldots \ldots . \ldots . \ldots 3^{\circ}$

12. C. rugosa $\ldots \ldots \ldots \ldots \ldots, 37^{\circ}-42^{\circ}$

C. intermedia $\ldots \ldots \ldots \ldots \quad 37^{\circ}$

C. salmanticensis $\ldots \ldots \ldots \quad 37^{\circ}$

19. C. lipolytica ........... $27^{\circ}-37^{\circ}$

20. C. zeylanoides .......... $32^{\circ}-36^{\circ}$

21. C. lipolytica var. deformans $32^{\circ}-34^{\circ}$

22. C. vartiovaarai ........... $32^{\circ}$

23. C. sake .............. $26^{\circ}-32^{\circ}$

24. C. humicola ........... $29^{\circ}$

\section{Commentaires}

Jusqu'en 1974 C. albicans était la levure la plus thermotolérante de toutes celles testées à ce jour. En janvier de cette année-là une souche de $C$. pseudotropicalis, cultivée à partir du contenu rectal d'une jeune Lionne, s'est développée jusqu'à $49^{\circ}$, soit $1^{\circ}$ centigrade au-dessus de la limite thermique supérieure de C. albicans.

En fonction de la valeur décroissante de l'espèce la plus thermotolérante du groupe, les micromycètes le plus couramment isolés à l'état parasitaire, saprophyte ou en survie de l'organisme humain et animal, s'ordonnent ainsi :
1. Aspergillus
2. Levures
3. Dermatophytes
4. Geotrichum
5. Scopulariopsis
6. Endomyces

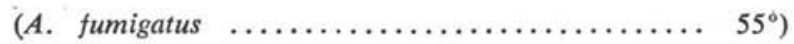

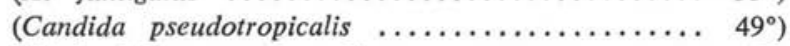

(Trichophyton mentagrophytes $\ldots \ldots \ldots \ldots \ldots \ldots, 43^{\circ}$ )

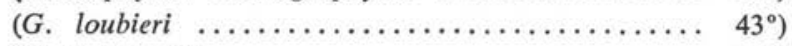

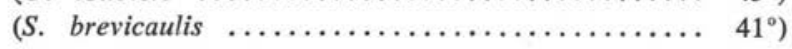

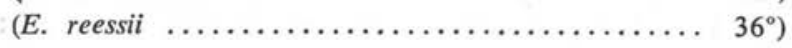


Les levures se situent donc à un haut niveau thermique. C'est parmi elles que se recrutent la plupart des constituants de la flore fongique et quelques agents pathogènes. Et c'est au genre Candida qu'appartiennent nombre de levures rencontrées dans des prélèvements d'origine humaine et animale.

C. pseudotropicalis, levure rarissime dans notre matériel, mis à part, les trois Candida thermophiles, $C$. albicans, $C$. krusei et $C$. tropicalis sont les représentants les plus habituels de la flore fongique digestive. Seul $C$. albicans, quoique signalé ici et là dans la nature, mérite la qualification de saprophyte obligatoire; lui seul, également passe le plus communément au parasitisme dans certaines circonstances. Les autres Candida, y compris ceux qui s'adaptent parfaitement à la vie dans le tube digestif, possèdent un habitat naturel ou artificiel (utilisation dans l'industrie alimentaire par exemple). C. bovina, qui fait maintenant partie des Torulopsis, et sa forme parfaite, Saccharomyces telluris, qui a pourtant été rencontrée pour la première fois dans le sol, doivent aussi être considérés comme des saprophytes obligatoires.

Comme le montre la liste ci-dessus, tous les Candida identifiés dans la pratique mycologique, médicale et vétérinaire, n’ont pas une température maximale compatible avec le saprophytisme, a fortiori le parasitisme, dans un organisme à sang chaud. A ce sujet, il est intéressant de citer l'exemple de $C$. zeylanoides, qui ne se multiplie plus à des niveaux thermiques relativement bas, et qui, cependant, a été rapporté comme agent pathogène. En se basant sur la température maximale et l'étude auxanographique d'un plus grand nombre de produits hydrocarbonés que ceux en usage dans la monographie des levures de 1952 (3), Van Uden et Farinha (13) ont différencié de C. zeylanoides une espèce plus thermotolérante, $C$. norvegensis (valeurs respectives, pour les auteurs, $30^{\circ}-32^{\circ}$ et $41^{\circ}-43^{\circ}$ ). Il est donc possible, soit que $C$. norvegensis ait été confondu avec $C$. zeylanoides, soit qu'il existe des souches de $C$. zeylanoides bien plus thermotolérantes que celles testées par Van Uden et Farihna, Van Uden et Buckley (in 4) et nous-mêmes. Au demeurant il s'agit d'espèces peu communes et, en ce qui concerne $C$. norvegensis, à pouvoir pathogène probablement occasionnel.

En étudiant la flore lévuriforme animale, une différenciation semblable à celle de C. zeylanoides et $C$. norvegensis s'était imposée à nous. Parmi les souches hébergées par nos hôtes, correspondant à la description de C. krusei de Lodder et Kreger-Van Rij (3), certaines, thermotolérantes, n'assimilaient pas le xylose (comme la souchetype de C. krusei, C.B.S. $\mathrm{n}^{\circ}$ 573, reçue de Delft), d'autres, à température maximale plus basse, utilisaient le xylose et furent nommées $C$. krusei var. transitoria (9). Notre souche-type ayant sporulé, la variété nouvelle décrite devenait un synonyme de $P$. fermentans (4), mais la description correspond à celle de C. lambica (4) qui, en 1952, sous le norn de Mycoderma lambica n'était encore qu'un synonyme de C. krusei (3).

Par la suite nous avons eu mainte fois l'occasion d'examiner des $C$. krusei et des C. lambica; nous avons constaté :

- qu'il existe toute une gamme dans l'assimilation du xylose, allant de la réaction précoce et fortement positive, à la réaction faiblement et tardivement posi- 
tive, enfin à la non-assimilation. Montrocher (5) rapporte une utilisation du xylose par une souche de $C$. krusei reçue de l'Institut Pasteur de Paris.

- qu'une souche n'assimilant pas le xylose (à identifier, classiquement, à C. krusei) ne poussait pas au-delà de $41^{\circ}$; tandis que trois souches assimilant faiblement le xylose (et à identifier à $C$. lambica) se développaient jusqu'à $41^{\circ}$.

Par conséquent, une distinction spécifique basée sur l'assimilation du xylose et la température maximale n'est plus possible, et la stimulation de la croissance de $C$. lambica par la thiamine nous paraît un caractère distinctif insuffisant. C. lambica devrait donc tomber en synonymie avec $C$. krusei.

La séparation entre $C$. sorbosa et $C$. krusei, reposant sur l'assimilation du sorbose et une température maximale de $37^{\circ}-40^{\circ}$ (in 4), de l'ordre de celle de C. lambica, nous semble également assez fragile. D'autant qu'une levure nommée Pichia kudriavzevii Boidin, Pignal et Besson (ex. Pichia (Issatchenkia) orientalis (Kudriavzev 1960) Kreger-Van Rij 1964) est tenue pour la forme parfaite à la fois de C. sorbosa et de C. krusei ; et que Kreger-Van Rij n'a pas observé de sporulation sur la souche-type, pas plus que Boidin et coll. (2) sur les 2 souches C.B.S. $\mathrm{n}^{\circ} 2911$ et 5459, ni nous-même sur la souche C.B.S. $\mathrm{n}^{\circ}$ 5459. Boidin et coll. écrivent à propos de $P$. kudriavzevii que, même en se rapportant à la figure originale de Kudriavzev il est difficile de préciser la position de cette espèce (2). Rappelons que $P$. fermentans est considéré comme la forme parfaite de C. lambica.

Pour toutes les levures du groupe C. krusei-P. fermentans, renfermant des souches dont la température maximale varie à l'intérieur d'un vaste intervalle de $35^{\circ}-47^{\circ}$, ou bien l'introduction de nouveaux critères aboutira à des distinctions taxonomiques plus tranchées, ou bien certaines entités actuellement reconnues tomberont en synonymie, peut-être même avec l'aide de ces nouveaux critères.

Dans le sang ingéré par une femelle d'Ixodes ricinus, parasite d'un Cerf d'Europe, Cervus elaphus, nous avons vu, à l'examen microscopique direct, un grand nombre de blastospores identifiées, en culture, à $C$. tropicalis (1). Beaucoup de levures ont déjà été signalées chez des Invertébrés, appartenant, fréquemment, à des espèces ascosporogènes; dans le tube digestif d'un Astacus pallipes nous avons également reconnu un Debaryomyces (kloeckeri) hansenii. Si l'on admet l'hypothèse séduisante selon laquelle les Candida parasites de l'homme, des mammifères et des oiseaux seraient des levures ascosporées ayant perdu leur reproduction sexuée par suite d'un parasitisme prolongé c'est, vraisemblablement, dans la nature que l'on retrouverait la forme ancestrale. Les travaux de Pitt et Miller (6), (7), et de Van der Walt (12) laissent espérer l'observation d'au moins une partie du cycle sexué in vitro. Selon l'hypothèse émise, les invertébrés terrestres et aquatiques joueraient un rôle de relais entre l'habitat naturel des Candida et les organismes à sang chaud. Il est à noter que chez les Invertébrés les levures peuvent être rencontrées : en survie, à l'état saprophyte, comme des microorganismes utiles pour certaines organogénèses de l'hôte et à l'état parasitaire. 


\section{Bibliographie}

1. ANDre (M.) et SAËZ (H.), 1964. - Sur la présence d'une levure, Candida tropicalis (Cast.), Berkhout chez Ixodes ricinus (L.). Ann. Méd. Vét., 108, 139-145.

2. Boidin (J.), Pignal (M.-C.) et Besson (M.), 1965. - Le genre Pichia sensu lato (Quatrième contribution). Bull. Sté. Mycol. France, 81, 566-606.

3. Lodder (J.) and Kreger-Van RiJ (N. J. W.), 1952, - The yeasts, A taxonomic study, 1 vol., North-Holland Cy. Publ., Amsterdam, 1385 p.

4. Lodder (J.), 1970, (Editor). - The yeasts. A taxonomic study, 1 vol., North-Holland Cy., Publ., Amsterdam-London, 713 p.

5. Montrocher (R.), 1967. - Les Candida à pouvoir fermentaire et n'assimilant pas les nitrates. Bull. Sté. Mycol. France, 83, 641-730.

6. PitT (J. I.) and Miller (M. W.), 1968. - Sporulation in Candida pulcherrima, Candida reukaufii and Chlamydozyma species: their relationships with Metschnikowia. Mycologia, 60, 663-685.

7. Pitt (J. I.) and Miller (M. W.), 1970. - The parasexual cycle in yeasts of the genus Metschnikowia. Mycologia, 62, 462-473.

8. Poncet (S.) et Arpin (M.), 1965. - Les Candida sans pouvoir fermentaire (Cryptococcacées). Ant. v. Leeuw., 31, 433-464.

9. SAËZ (H.), 1965. - Candida krusei (Castellani) Berkhout var. transitoria, nouvelle variété de levure isolée chez divers mammifères et oiseaux. Bull. Sté. Linn. Lyon, $34,265-270$.

10. SAËZ (H.), 1966. - Quelques caractéristiques physio-morpholiques de Candida albicans et incidence du facteur température sur les levures. Ann. Méd. Vét. 6, 448-458.

11. SAËz (H.), 1973. - Candida nouvelii, nouvelle espèce de levure rencontrée chez deux mammifères sauvages morts en captivité. Bull. Sté. Mycol. France, 89, 79-82.

12. VAN DER WALt (J.P.), 1970. - The genus Syringospora Quinquad emend. Mycopath. Mycol. Appl., 40, 231-243.

13. VAN Uden (N.) and FARINHA (M.), 1958. - On the significance of temperature relations and vitamin deficiency patterns in the delimitation of yeast species. Portug. Acta Biol., 6, 161-178.

14. Van Uden (N.) and Do Carmo-Sousa (L.), 1959. - Further studies on the significance of temperature relations and deficiency patterns in yeast taxonomy. Portug. Acta Biol., 6, 239-256.

15. Van Uden (N.) and Do Carmo-Sousa (L.), 1957. - Yeasts from the bovine caecum. J. Gen. Microbiol., 16, 385-395. 